\title{
Dynamic Routing For Flying Ad Hoc Networks
}

\author{
K.G. Aarthi ${ }^{1}$, Dr. K. Santhi ${ }^{2}$ \\ Research Scholar, Department of Computer Science, Sri Ramakrishna College of Arts and Science for Women, \\ Coimbatore, India ${ }^{1}$ \\ Associate Professor, Department of Computer Science, Sri Ramakrishna College of Arts and Science for Women, \\ Coimbatore, India ${ }^{2}$
}

\begin{abstract}
The conventional border patrol systems suffer from intensive human involvement. Recently, unmanned border patrol systems employ high-tech devices, such as unmanned aerial vehicles (UAVs), unattended ground sensors, and surveillance towers equipped with camera sensors. These nodes rebroadcast the received beacon until it reaches all UAVs in the network. During their propagation, when passing by the UAVs, the beacons carry the identification of the UAVs that relay them. Like this, when a UAVs receives a beacon, it is able to update the list of its neighbors and the distance, in number of hops, to the tail node. The proposed algorithm FANET is used to improve the packet delivery and reduce delay time between sources to destination for UAVs network. In this approach, a node maintains the topology information involving its one-hop neighbors. During a reconfiguration process following a path break, improve FANET protocol has the unique property to limit the control packets to a small region of network. The implementation of FANET routing protocols in UAVs networks serves to fulfil the purpose of reservation of sufficient resources along a route so as to meet the QoS requirements of a flow. The FANET can efficiently calculate the communication delay of a packet across an UAVs network is the path selection based on score value and less delay path only selected to send by a packet to reach the destination from the source. The experimental result using NS2.34 simulator and compare the end-to-end latency of a packet at the network layer are processing delay, packetization delay, transmission delay, queuing delay and the propagation delay.
\end{abstract}

Keywords: Unmanned Aerial Vehicles (UAVs), Flying Ad Hoc Networks (FANET), Optimized Link State Routing Protocol (OLSR), Traffic Aware Routing (TAR).

\section{INTRODUCTION}

Unmanned Aerial Vehicles (UAVs) are considered a promising alternative to piloted aircrafts both in civil and military applications. These vehicles are especially useful in dangerous missions where human lives might be put at risk or even in dull tasks where most of the functionalities of a human pilot can be automated. There is a wide range of potential applications in which UAVs can substantially improve the efficiency and costs of the mission such as border surveillance, search and rescue, environmental monitoring and military tactics to name a few.

During the last century, there has been a remarkable research effort to investigate new ways of endowing UAS with higher levels of autonomy and to properly manage the inevitable changes generated in their operability. This effort has been clearly led by United States with an ambitious investment of money and resources in this technology. The rapid evolution in the performance of unmanned vehicles has not always been accompanied by the required technical analysis of the maturity and efficiency of the algorithms and technologies developed. Therefore, proper test and validation campaigns are necessary to consolidate the latest advances in the field of automation and autonomy.

FANETs are a special case of mobile ad hoc networks (MANETs) characterized by a high degree of mobility. In a FANET, the topology of the network can change more frequently than in a typical MANET or vehicle ad hoc network (VANET). As a consequence, the network routing becomes a crucial task. The network routing algorithms, which have been designed for MANETs, such as BABEL or the optimized link-state routing (OLSR) protocol, fail to follow the evolution of the network topology. It is possible to bypass this problem by considering star networks with static routing. However, star architectures restrict the operative area of groups of UAVs, because the nodes cannot fly out of the communication range of the control center.

Emergency Response Communication is a component of communication focusing on the communication between emergency responders during emergency situations. The main categories of emergency responders can be classified as Fire and Rescue, Emergency Medical Services (EMS), and Police. Each of these services focus on different tasks during an emergency and currently use their own wireless communication frequencies and channels.

UAVs are designed to replace humans in tasks that are considered too" dull, dirty, or dangerous". As improvements in technology have made IC chips and other equipment smaller, UAVs have become more suited to nimbler and more precise tasks. The microcontroller is the brains of the machine; it processes inputs and sends the appropriate signals as 
outputs to maintain and control flight. Brushless DC electric motors are typically used with UAVs as their power-toweight ratio is complimentary to the weight requirements needed to achieve flight.

The border patrol systems suffer from intensive human involvement. Recently, unmanned border patrol systems employ high-tech devices, such as unmanned aerial vehicles, unattended ground sensors, and surveillance towers equipped with camera sensors. In this proposed system to improve the packet delivery based on packet delay in the path and traffic on the path going to calculate. The proposed work can efficiently calculate the communication delay of a packet across an UAVs network is the path selection based on score value and less delay path only selected to send by a packet to reach the destination from the source

\section{LITERATURE REVIEW}

$>\quad$ C. Barrado et al., [1] Forest fires are a challenging problem for many countries. They often cause economical lost and ecological damage, and they can sometimes even cost human lives. Finding hot spots immediately after a fire is an important part of fighting forest fires. The main objective is to obtain a temperature map of the burned area, to locate the most critical embers. This information can help fire fighter managers make the correct decisions about ground crew movements. The main objective is to obtain the area's temperature map to detect the critical hot spots. An aerial remote-sensing resource takes infrared (IR) images to obtain this temperature map. The second objective is to take visual photographs of the hot spots' surroundings for their best location. Important requirements include:

- Rapidness. The information should be available to fire managers as soon as possible.

- Confidence. The information should help managers make the correct decisions about ground crew movements or even about when to abandon the area.

- Cost. The overall task's cost should be reduced.

$>\quad$ Z. Sun et al., [2] The conventional border patrol systems suffer from intensive human involvement. Recently, unmanned border patrol systems employ high-tech devices, such as unmanned aerial vehicles, unattended ground sensors, and surveillance towers equipped with camera sensors. However, any single technique encounters inextricable problems, such as high false alarm rate and line-of-sight-constraints. There lacks a coherent system that coordinates various technologies to improve the system accuracy. sensor networks

$>\quad$ Rubin and R. Zhang [3] Unmanned aerial vehicles (UAVs) have been used for surveillance and reconnaissance operations. Such communications enabled platforms can also be effectively utilized to enhance the communications transport capabilities of a mobile ad hoc wireless network. When properly embedded into the architecture of a communications network, the resulting UAV aided terrestrial network architecture is expended into a multi-layered hierarchical network structure. Robust service is critically required for supporting applications that involve flow transactions that must not, with high probability, be transported along routes that are prematurely interrupted.

$>\quad$ E. P. de Freitas et al., [4] An important problem in Wireless Sensor Networks (WSN) is the occurrence of failures that lead to the disconnection of parts of the network, compromising the final results achieved by the WSN operation [4]. A way to overcome such problem is to provide a reliable connection to support the connectivity via other types of nodes that communicate with the sensor nodes. This paper proposes the usage of a network composed by Unmanned Aerial Vehicles (UAVs) as a relay network to guarantee the delivery of data produced by WSN nodes on the ground to the users. Results from simulations of the proposed technique are provided and discussed.

$>\quad$ F. Jiang and A. Swindlehurst [5] In this paper we consider a collection of mobile single-antenna ground nodes communicating with a multi-antenna unmanned aerial vehicle (UAV) on a multiple-access ground-to air wireless communications link. The UAV uses beam forming to mitigate the inter-user interference and achieve spatial division multiple access (SDMA). In addition, the UAV dynamically adjusts its heading in order to maximize a lower bound on either the ergodic sum rate of the uplink channel or the minimum ergodic rate of the worst-case user, using a Kalman filter to track the positions of the mobile ground nodes.

> I. Bekmezci, et., al., [6] One of the most important design problems for multi-UAV (Unmanned Air Vehicle) systems is the communication which is crucial for cooperation and collaboration between the UAVs. If all UAVs are directly connected to an infrastructure, such as a ground base or a satellite, the communication between UAVs can be realized through the infra structure. The differences between FANETs, MANETs (Mobile Ad-hoc Networks) and VANETs (Vehicle Ad-Hoc Networks) are clarified first, and then the main FANET design challenges are introduced. Along with the existing FANET protocols, open research issues are also discussed.

$>\quad$ O. Sahingoz, et., al., [7] With the advances in computation, sensor, communication and networking technologies, utilization of Unmanned Aerial Vehicles (UAVs) for military and civilian areas has become extremely popular for the last two decades. Since small UAVs are relatively cheap, the focus is changing, and usage of several small UAVs is preferred rather than one large UAV.

$>\quad$ K. Zhang, et., al., [8] This paper introduces the usage and development of mobile Ad Hoc UAV (Unmanned Aerial Vehicles) network firstly, then, brings forward some problems of Ad Hoc UAV network presently, and gives 
some related research topics about mobile Ad Hoc UAV fleet network, especially routing protocol and date integrity technologies [8]. Finally, give a conclusion about our preliminary studies and this paper. QoS definition is "The collective effect of service performance which determines the degree of satisfaction of a user of a service".

\section{PREDICTIVE OLSR (EXISTING)}

\subsection{INTRODUCTION}

As pointed out, FANETs are a special case of mobile ad hoc networks (MANETs) characterized by a high degree of mobility. In a FANET, the topology of the network can change more frequently than in a typical MANET or vehicle ad hoc network (VANET). As a consequence, the network routing becomes a crucial task. The network routing algorithms, which have been designed for MANETs, such as BABEL or the optimized link-state routing (OLSR) protocol fail to follow the evolution of the network topology. It is possible to bypass this problem by considering star networks with static routing. However, star architectures restrict the operative area of groups of UAVs, because the nodes cannot fly out of the communication range of the control centre. In this paper, we focus on partially connected mesh ad hoc networks that enable the UAVs to use multi-hop communication to extend the operative area. In this case, the problem of highly dynamic routing must be faced.

\subsection{PREDICTIVE OLSR}

The Predictive OLSR used systems are listed below:

$>\quad$ Optimized link-state routing

$>\quad$ The Babel routing protocol

$>\quad$ Speed-aware routing for UAV Ad-Hoc networks

\subsubsection{Optimized link-state routing}

The Optimized Link State Routing (OLSR) protocol for mobile ad hoc networks. The protocol is an optimization of the classical link state algorithm tailored to the requirements of a mobile wireless LAN. The key concept used in the protocol is that of multipoint relays (MPRs). MPRs are selected nodes which forward broadcast messages during the flooding process. This technique substantially reduces the message overhead as compared to a classical flooding mechanism, where every node retransmits each message when it receives the first copy of the message. In OLSR, link state information is generated only by nodes elected as MPRs. Thus, a second optimization is achieved by minimizing the number of control messages flooded in the network. As a third optimization, an MPR node may choose to report only links between itself and its MPR selectors. Hence, as contrary to the classic link state algorithm, partial link state information is distributed in the network. This information is then used for route calculation. OLSR provides optimal routes (in terms of number of hops).

\subsubsection{Babel routing protocol}

Babel is a loop-avoiding distance-vector routing protocol that is robust and efficient both in ordinary wired networks and in wireless mesh networks. More precisely, Babel has the following properties:

$>\quad$ When every prefix is originated by at most one router, Babel never suffers from routing loops;

$>\quad$ When a prefix is originated by multiple routers, Babel may occasionally create a transient routing loop for this particular prefix; this loop disappears in a time proportional to its diameter, and never again (up to an arbitrary garbagecollection (GC) time) will the routers involved participate in a routing loop for the same prefix;

$>\quad$ Assuming reasonable packet loss rates, any routing black-holes that may appear after a mobility event are corrected in a time at most proportional to the network's diameter.

Babel has provisions for link quality estimation and for fairly arbitrary metrics. When configured suitably, Babel can implement shortest-path routing, or it may use a metric based, for example, on measured packet loss. Babel nodes will successfully establish an association even when they are configured with different parameters. For example, a mobile node that is low on battery may choose to use larger time constants (hello and update intervals, etc.) than a node that has access to wall power. Conversely, a node that detects high levels of mobility may choose to use smaller time constants. The ability to build such heterogeneous networks makes Babel particularly adapted to the wireless environment. Finally, Babel is a hybrid routing protocol, in the sense that it can carry routes for multiple network-layer protocols (IPv4 and IPv6), whichever protocol the Babel packets are themselves being carried over.

\subsubsection{Speed-aware routing}

Predictive-OLSR an extension to the Optimized Link-State Routing (OLSR) protocol: it enables efficient routing in very dynamic conditions. The key idea is to exploit GPS information to aid the routing protocol. Predictive-OLSR weights the expected transmission count (ETX) metric, taking into account the relative speed between the nodes. We 
provide numerical results obtained by a MAC-layer emulator that integrates a flight simulator to reproduce realistic flight conditions. These numerical results show that Predictive-OLSR significantly outperforms OLSR and BABEL, providing a reliable communication even in very dynamic conditions.

\subsection{ISSUES}

$>\quad$ OLSR Protocol fails to provide a reliable communication [10].

$>\quad$ Fail to follow the evolution of the network topology [2].

$>\quad$ The nodes cannot fly out of the communication range of the control center cannot send the message [7].

$>\quad$ More delay of packet sending to the tail node [14].

$>\quad$ Packet loss due to high mobility [6].

$>\quad$ Fast-moving nodes are a small percentage of packet delivery [15]

$>\quad$ The additional overhead of control packets [5].

\section{FANET-FLYING AD HOC NETWORKS (PROPOSED)}

\subsection{INTRODUCTION}

The advent of commercial drones or unmanned aerial vehicles (UAVs) is expected to facilitate the deployment of a plethora of UAVs-based applications. This new routing protocol relies on choosing, at each moment and ahead of time, the most connected path among others available and avoid available paths that can be quickly broken. Connectivity paths are done and undone due to the high mobility of the vehicles and to the encountered obstructions and obstacles. A path is considered most connected based on the traffic density and the connectivity of the vehicles within a road segment. A path connectedness is measured through (1) periodic Hello messages exchanged between vehicles, or (2) by forwarding the data packets directly to UAVs within range when there are no available routing paths and then forward the data packet directly to the destination if it is within the transmission range of the UAV, or (3) to the vehicle located at the most appropriate intersections where there are available connected road segments leading to the destination. This scheme overcomes the presence of obstacles when calculating the traffic density, connectivity and the packets delivering.

\subsection{UAVS NETWORK MODEL}

The main objectives of this thesis are to:

$>$ Develop an adaptive connectivity and traffic aware algorithm for UAVs network.

$>$ Analyze goodput and energy efficiency for an UAVs network.

$>$ Evaluate the effect of reuse distance and signal to interference plus noise ratio (SINR) on an UAVs network.

$>$ Investigate security concerns within an UAVs network.

\subsection{CONNECTIVITY ANALYSIS}

$>\quad$ In an UAVs network, once two UAVs are within the communication range, whether or not they are reachable after certain time $t$ can be checked by using their velocities, their accelerations and the time interval. For a given UAV with its initial velocity $\vec{v}(0)$, the instantaneous velocity $\vec{v}(t)$ at time $t$ is defined as

$$
\vec{v}(t)=\vec{v}(0)+\int_{0}^{t} a(y) d y
$$

where $\mathrm{a}(\mathrm{y})$ is the acceleration of a UAV at time $\mathrm{y}$. Using (2.1), the distance travelled by a UAV for an interval [0; $\mathrm{t}]$ is

$$
D(t)=\int_{0}^{t} \vec{v}(y) d y
$$

Thus, using (2) for a time interval [0; t], the distances travelled by any UAV can be calculated. Consider $\mathrm{i}$ and $\mathrm{j}$ UAVs calculate $\mathrm{Di}(\mathrm{t})$ and $\mathrm{Dj}(\mathrm{t})$ using (1). Then the distance between the UAVs $i$ and $\mathrm{j}$ for the interval [0; t], where UAV i is following $\mathrm{j}$ and initial separation distance was $\mathrm{z}$, is given by

$$
D_{e}=I(i, j)\left[D_{i}(t)-D_{j}(t)\right], \quad I(i, j) \in\{1,-1\}
$$

where if $\operatorname{Di}(t)>\operatorname{Dj}(t), I(i ; j)=-1$, otherwise $I(i ; j)=1$. Note that the distance travelled by the ground/base station $\mathrm{D}(\mathrm{t})=0$ as it is fixed. After time t, UAVs to be able to reach wirelessly and UAVs not to crash with others, the following condition (for both longitude and latitude) should be satisfied

$$
\mathrm{S}_{\mathrm{d}} \leq \mathrm{D}_{\mathrm{e}} \leq \min [\mathrm{m}(\mathrm{R}(\mathrm{n})\} \forall \mathrm{n} \leq \overline{\mathrm{R}}
$$

where $\mathrm{Sd}$ is the safety separation distance between UAVs. The transmission range R can be computed as 


$$
R=\sqrt{\frac{P_{t} G}{P_{r}}} \quad \frac{c}{4 \pi f}
$$

where $\mathrm{Pt}$ is transmitting power, $\mathrm{G}$ is an effective gain, $\mathrm{Pr}$ is received power, $\mathrm{c}=3108 \mathrm{~m}=\mathrm{s}$ is velocity of light, and $\mathrm{f}$ is frequency used to communicate. Maximum allowed transmission power (i.e., $\mathrm{Pt}=\mathrm{Pmax}$ ) for a given band $\mathrm{f}$ can be used to calculate the maximum transmission range R. If Sd De in (4) is not satisfied, UAVs must repel each other, otherwise they are close enough to crash into each other. If $\mathrm{De}_{-} \operatorname{minffR}(\mathrm{n}) \mathrm{g} 8 \mathrm{ng}$ _ $\mathrm{R}$ is not satisfied, UAVs would not be able to communicate with each other using single-hop communication. In both cases, UAVs change their directions and/or speeds to avoid a collision and maintain a communication link with each other. In this case, UAVs n computes resultant vector by adding a unit vector in direction $n(t)$ to unit vector ${ }^{\vec{v}} \mathrm{vn}(\mathrm{t}) \mathrm{jj} \mathrm{jn}(\mathrm{t}) \mathrm{jj}$ and the angle of the resultant vector is the new direction of a given UAV n, n $(\mathrm{t}+1)$ based on the angle of arrival. Each UAV determines the new direction based on current position of the UAV and angle of arrival from other UAVs.

\section{Algorithm 1 Connectivity for UAVs}

$>$ Input: initial velocities $\overrightarrow{\mathrm{vn}}(0)$, maximum allowed transmit power Pmax, current direction $\mathrm{n}(\mathrm{t})$, and safety distance Sd.

$>$ Output: Adapted transmission range, new direction of $U A V_{n}:-n(t+1)$, and the probability of successful connectivity

1: Initialize a counter: counter $=0$;

2: For each sensing time interval, $t$ do

3: For each $\mathrm{UAV}_{\mathrm{n}}$ do

4: if $\mathrm{UAV}_{\mathrm{n}}$ is connected to base station (BS) by single-hop (i.e., condition (4) is satisfied) then

5: Estimate the new location after time $t$ basedon the current velocity and transmits range.

6: if $U_{A} V_{n}$ will still be connected to BS based on estimated location in step 7, then continue with the current settings for $\mathrm{UAV}_{\mathrm{n}}$.Increment the counter by 1 .

7: Otherwise Adapt the transmission range and change the direction of a given UAV, towards BS with the help of angle of arrival, and GOTO Step 6 end if.

8: Else if $U_{A} V_{n}$ is not connected to $B S$ then

9:Check if it has neighboring UAVs. If not, adapt transmission range and check if it can reach other UAVs. If yes, continue with existing settings and increment the counter by 1 .

10: Check if at least one neighbor exists, estimate location and check if it is reachable after $t$ time. If not, change the direction and transmit range, and GOTO step 10 end if.

11: end for

12: Successful probability $P_{s}=\frac{\text { Counter }}{N}$

13: end for

\subsection{FANET USING UAVs}

This protocol is to find the shortest and the most reliable segment among others available beforehand based on a score calculated permanently by the vehicles situated at the intersections for all the segments around. In addition, the UAVs have a global vision of all the intersections surrounding them (in range). Consequently, the UAVs can immediately deduct the most connected segment based on the best scores given at each intersection which are shared through the periodical exchange of Hello messages by the vehicles located at the intersections. The can select UAVs as forwarding node instead of road segment in the case where the UAVs detect a score on another intersection better than calculated by another UAVs located at the current intersection.

\section{Traffic aware Routing Implementation algorithm}

Step 1: $C \leftarrow$ The current UAV;

Step 2: D $\leftarrow$ the destination UAV;

Step 3: $\mathrm{Nc} \leftarrow$ The set of one hop neighbors of $\mathrm{C}$;

Step 4: $\mathrm{J} \leftarrow$ The current intersection;

Step 5: if $C=D$ then

Step 6: Received packet (Success);

Step 7: else

Step 8: if $D \in N c$ then

Step 9: Forward (packet,D); 
Step 10: else

Step 11: if Position $(C) \in$ Intersection areas then

Step 12: for each Segment i do

Step 13:

Step 14: J $\leftarrow$ Max of all (Segmenti, Scorei);

$$
\text { Score }_{\mathrm{i}}=\frac{\text { TOTAL }_{\mathrm{UAV}}}{\mathrm{D}_{\mathrm{w}}} \cdot\left(\mathrm{L}_{\mathrm{i}} \times \mathrm{R}_{\mathrm{j}}\right)
$$

Step 15: // Select the next intersection.

Step 16: if $J \geq S C$ then

Step 17: SFS $\leftarrow$ UAV;

Step 18: packet Forwarding (packet, SFS) to the next

Step 19: // SC is the best score obtained by the UAV.

\section{RESULTS AND DISCUSSION}

\subsection{INTRODUCTION NS2}

Network Simulator (Version 2), widely known as NS2, is simply an event-driven simulation tool that has proved useful in studying the dynamic nature of communication networks. Simulation of wired as well as wireless network functions and protocols (e.g., routing algorithms, TCP, UDP) can be done using NS2. In general, NS2 provides users with a way of specifying such network protocols and simulating their corresponding behaviors.NS2 consists of two key languages: CCC and Object-oriented Tool Command Language $(\mathrm{OTcl})$. While the CCC defines the internal mechanism (i.e., a backend) of the simulation, the OTcl sets up simulation by assembling and configuring the objects as well as scheduling discrete events (i.e., a frontend). The CCC and the OTcl are linked together using TclCL.

After simulation, NS2 outputs either text-based simulation results. To interpret these results graphically and interactively, tools such as NAM (Network AniMator) and XGraph are used. To analyze a particular behavior of the network, users can extract a relevant subset of text-based data and transform it to a more conceivable presentation.

Table 5.1 Simulation Environment used for the FANET

\begin{tabular}{|l|l|}
\hline \multicolumn{1}{|c|}{ Parameters } & \multicolumn{1}{c|}{ Values } \\
\hline Simulator & NS2.34 \\
\hline Routing Protocol & OLSR \\
\hline Experiment Area & $650 \mathrm{~m} * 250 \mathrm{~m}$ \\
\hline UAV Node Deployment & Random \\
\hline No of Nodes & 20 \\
\hline Node Speed & $50 \sim 100 \mathrm{~m} / \mathrm{s}$ \\
\hline Type of Transmission & CBR \\
\hline MAC Layer & Mac/802_11 \\
\hline Transmission Range & $250 \mathrm{~m}$ \\
\hline Simulation Time & $600 \mathrm{sec}$ \\
\hline Packet Size & $512 \mathrm{kbps}$ \\
\hline Pause Time & $10 \mathrm{sec}$ \\
\hline
\end{tabular}

\subsection{PERFORMANCE METRICS}

\section{Packet Delivery Success Rate}

The ratio of the data packets delivered to the destinations to those generated by the CBR sources is known as packet delivery fraction.

$$
\text { PDR }=\frac{\sum_{\mathrm{i}} \text { Packets Delivered }}{\sum_{\mathrm{i}} \text { Packets Sent }}
$$

\section{Average latency}

The speed of light imposes a minimum propagation time on all electromagnetic signals. It is not possible to reduce the latency below

$$
\mathrm{t}=\frac{\mathrm{S}}{\mathrm{C}_{\mathrm{m}}}
$$

where $\mathrm{s}$ is the distance and $\mathrm{cm}$ is the speed of light in the medium. This approximately means 1 extra millisecond RTT for $100 \mathrm{~km} / 62$ miles of distance between hosts. Other delays also occur in intermediate nodes. In packet switched networks delays can occur due to queueing 


\section{$>\quad$ Routing Overhead}

Normalized Routing Load (or Normalized Routing Overhead) is defined as the total number of routing packet transmitted per data packet. It is calculated by dividing the total number of routing packets sent (includes forwarded routing packets as well) by the total number of data packets received.

Routing Overhead

$$
=\frac{\text { total number of packet transmitted }}{\text { number of routing packets sent }} \times \text { total number of data packets received }
$$

\section{Average Outage Tim0065}

The network performance in terms of average outage time. The outage time of a run is the sum of all the outage durations.

$$
\text { Outage }=\frac{\text { sending node ratio range }- \text { reciving node ratio range }}{\text { Packet Sent }}
$$

\section{$>\quad$ Average Goodput}

In computer networks, goodput is the application-level throughput (i.e. the number of useful information bits delivered by the network to a certain destination per unit of time). The amount of data considered excludes protocol overhead bits as well as retransmitted data packets.

$$
\text { Goodput }=\frac{(\text { max no of pkts recvd by the rx in sequence }}{\text { total number of pkts sent by the sender including retransmissions }}
$$

\section{Throughput}

Throughput is the amount of total number of packets delivered over the total simulation time.

$$
\text { Throughput }=\frac{\text { total packet recived }}{\text { total packet sent }}
$$

\section{End-to-End Delay}

The average time taken by a data packet to arrive in the destination. It also includes the delay caused by route discovery process and the queue in data packet transmission. Only the data packets that successfully delivered to destinations that counted.

$$
\text { EE Delay }=\frac{\Sigma \text { arrive time }- \text { send time }}{\Sigma \text { Number of connections }}
$$

\subsection{COMPARISION GRAPH}

Average Goodput (ms)

\begin{tabular}{|l|c|c|c|c|c|c|c|c|c|c|}
\hline \multirow{2}{*}{ Protocol } & \multicolumn{10}{|c|}{ Hello Interval } \\
\cline { 2 - 11 } & $\mathbf{0 . 2}$ & $\mathbf{0 . 4}$ & $\mathbf{0 . 6}$ & $\mathbf{0 . 8}$ & $\mathbf{1}$ & $\mathbf{1 . 2}$ & $\mathbf{1 . 4}$ & $\mathbf{1 . 6}$ & $\mathbf{1 . 8}$ & $\mathbf{2 . 0}$ \\
\hline P-OLSR & 0.1 & 0.3 & 0.5 & 1.1 & 1.4 & 1.7 & 1.9 & 2.0 & 2.1 & 2.4 \\
\hline FANET & 0.2 & 0.5 & 0.8 & 1.5 & 1.8 & 1.9 & 2.0 & 2.1 & 2.3 & 2.6 \\
\hline
\end{tabular}

Table 5.1 Hello Interval of Node vs Goodput

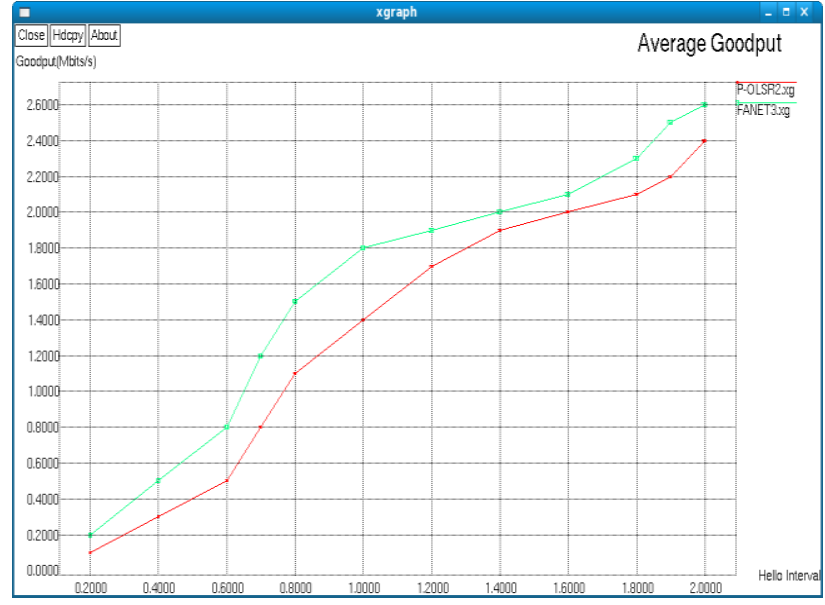

Figure 5.1 Hello Interval of Node vs Goodput 
Fig. 5.1 show the output of average goodput comparison in hello interval time based taken in average of all the node received mbit/s in FANET give high goodput compare with Predictive OLSR.

\section{Throughput (kbps)}

\begin{tabular}{|l|c|c|c|c|c|c|c|c|c|}
\hline \multirow{3}{*}{ Protocol } & \multicolumn{10}{|c|}{ No of Nodes (n) } \\
\cline { 2 - 11 } & $\mathbf{2}$ & $\mathbf{4}$ & $\mathbf{6}$ & $\mathbf{8}$ & $\mathbf{1 0}$ & $\mathbf{1 2}$ & $\mathbf{1 4}$ & $\mathbf{1 6}$ & $\mathbf{1 8}$ \\
\hline P-OLSR & 2.1 & 2.4 & 3.5 & 4.2 & 4.7 & 5.1 & 5.5 & 6.1 & 6.6 \\
\hline FANET & 3.8 & 4.1 & 4.8 & 5.5 & 5.9 & 6.3 & 6.8 & 7.5 & 7.8 \\
\hline
\end{tabular}

Table 5.2 No of node vs Throughput (kbps)

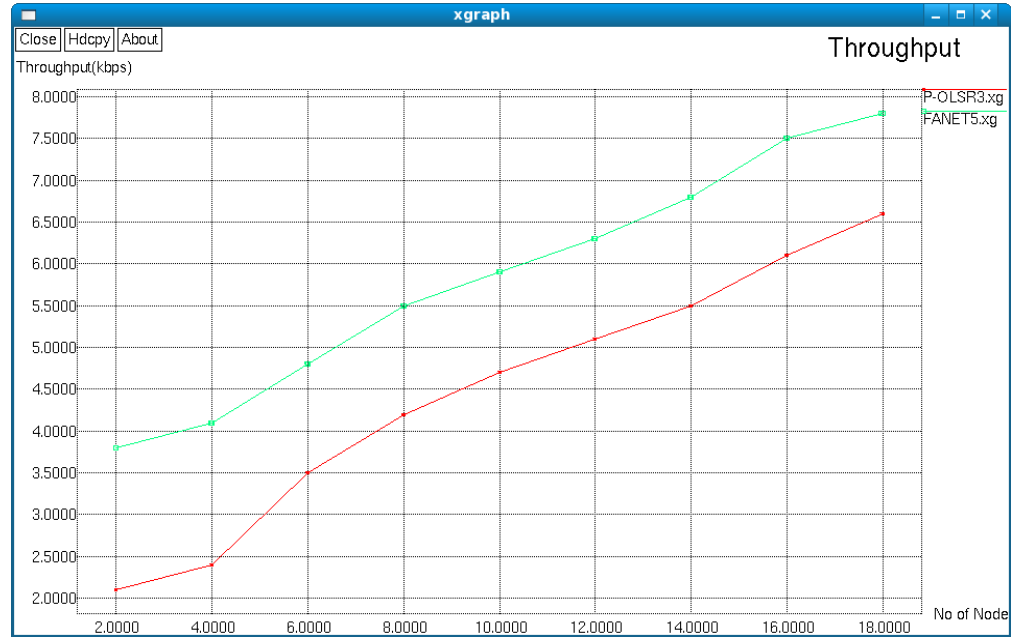

Figure 5.2 No of node vs Throughput (kbps)

Fig. 5.2 show the output of throughput comparison in no of node based taken in every node received kbps in the FANET give high throughput compare with Predictive OLSR.

Goodput (mbits/s)

\begin{tabular}{|l|c|c|c|c|c|c|c|c|c|}
\hline \multirow{2}{*}{ Protocol } & \multicolumn{10}{|c|}{ Simulation Time(s) } \\
\cline { 2 - 10 } & $\mathbf{1 0}$ & $\mathbf{5 0}$ & $\mathbf{1 0 0}$ & $\mathbf{1 5 0}$ & $\mathbf{2 0 0}$ & $\mathbf{2 5 0}$ & $\mathbf{3 0 0}$ & $\mathbf{3 5 0}$ & $\mathbf{4 0 0}$ \\
\hline P-OLSR & 0.261 & 0.366 & 0.462 & 0.560 & 0.663 & 0.751 & 0.863 & 0.962 & 1.156 \\
\hline FANET & 0.463 & 0.563 & 0.662 & 0.760 & 0.864 & 0.958 & 1.163 & 1.264 & 1.353 \\
\hline
\end{tabular}

Table 5.3 Simulation time vs Goodput (mbits/s)

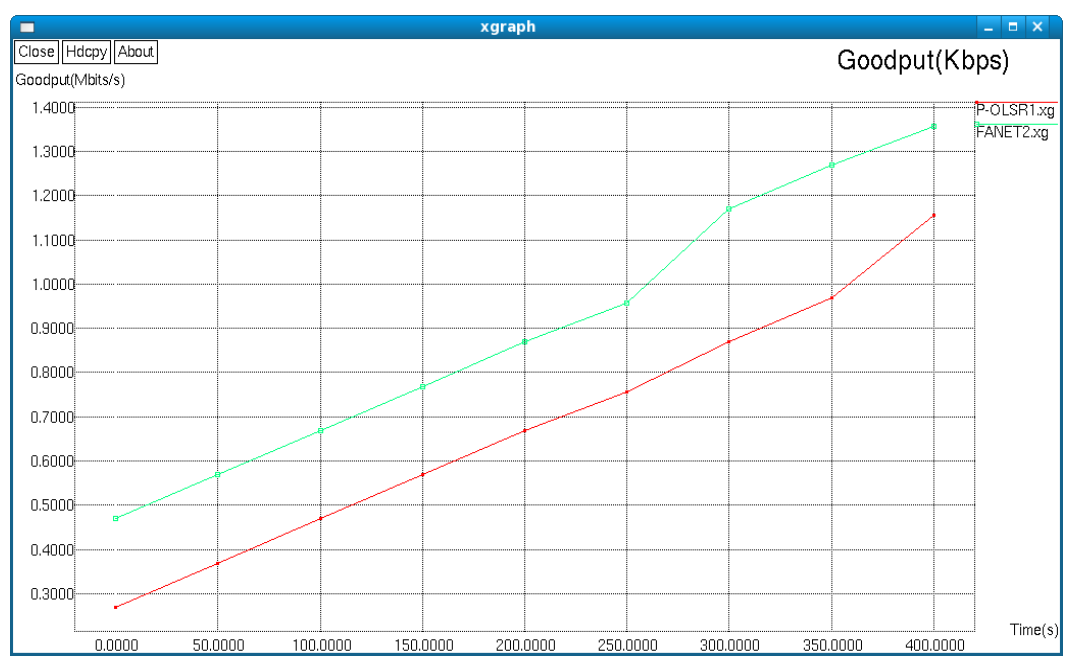

Fig 5.3 Simulation time vs Goodput(mbits/s)

Fig. 5.3 show the output of goodput comparison in hello interval time based taken in each and every node received $\mathrm{mbit} / \mathrm{s}$ in the FANET give high goodput compare with Predictive OLSR. 
Packet Delivery Ratio (\%)

\begin{tabular}{|l|c|c|c|c|c|c|c|c|c|}
\hline \multirow{2}{*}{ Protocol } & \multicolumn{10}{|c|}{ No of Nodes (n) } \\
\cline { 2 - 11 } & $\mathbf{2}$ & $\mathbf{4}$ & $\mathbf{6}$ & $\mathbf{8}$ & $\mathbf{1 0}$ & $\mathbf{1 2}$ & $\mathbf{1 4}$ & $\mathbf{1 6}$ & $\mathbf{1 8}$ \\
\hline P-OLSR & 54 & 50 & 64 & 79 & 81 & 85 & 87 & 89 & 91 \\
\hline FANET & 65 & 67 & 64 & 85 & 86 & 87 & 91 & 95 & 96 \\
\hline
\end{tabular}

Table 5.4 No of node vs Packet delivery ratio $(\%)$

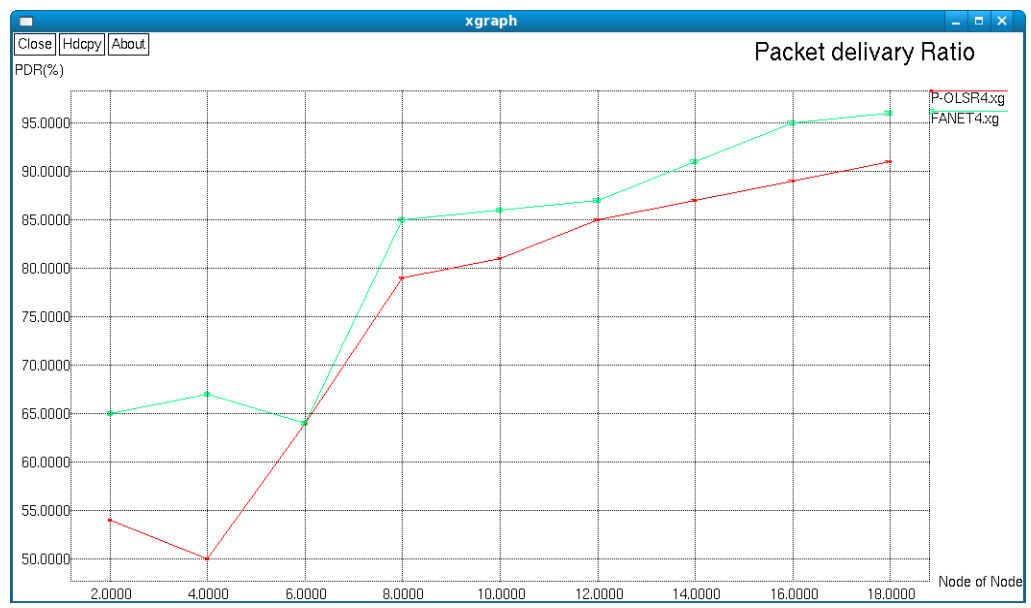

Figure 5.4 No of node vs Packet delivery ratio (\%)

Fig. 5.4 show the output of PDR comparison in no of node vs PDR. The FANET give high PDR compare with Predictive OLSR.

End-To-End Delay

\begin{tabular}{|l|c|c|c|c|c|c|c|c|c|}
\hline \multirow{2}{*}{ Protocol } & \multicolumn{10}{|c|}{ No of Nodes (n) } \\
\cline { 2 - 10 } & $\mathbf{2}$ & $\mathbf{4}$ & $\mathbf{6}$ & $\mathbf{8}$ & $\mathbf{1 0}$ & $\mathbf{1 2}$ & $\mathbf{1 4}$ & $\mathbf{1 6}$ & $\mathbf{1 8}$ \\
\hline P-OLSR & 5.8 & 4.1 & 3.2 & 3.1 & 2.8 & 2.5 & 2.4 & 2.1 & 1.9 \\
\hline FANET & 4.1 & 3.2 & 2.7 & 2.1 & 1.9 & 1.2 & 1.1 & 1.0 & 0.9 \\
\hline
\end{tabular}

Table 5.5 Source to destination vs End-To-End Delay

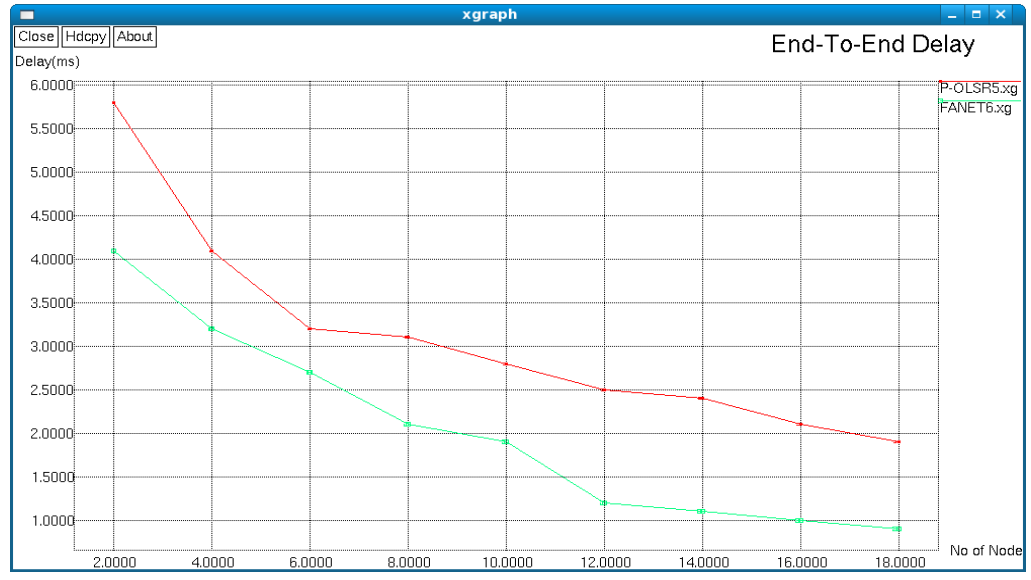

Figure 5.5 Source to destination vs End-To-End Delay

Fig. 5.5 show the output of end-to-end Delay comparison in no of node vs delay time in mile second. The FANET give low time based packet sending compare with Predictive OLSR.

Average Outage Time (s)

\begin{tabular}{|l|c|c|c|c|c|c|c|c|c|c|}
\hline \multirow{3}{*}{ Protocol } & \multicolumn{10}{|c|}{ Hello Interval } \\
\cline { 2 - 11 } & $\mathbf{0 . 2}$ & $\mathbf{0 . 4}$ & $\mathbf{0 . 6}$ & $\mathbf{0 . 8}$ & $\mathbf{1 . 0}$ & $\mathbf{1 . 2}$ & $\mathbf{1 . 4}$ & $\mathbf{1 . 6}$ & $\mathbf{1 . 8}$ & $\mathbf{2 . 0}$ \\
\hline P-OLSR & 5.1 & 5.5 & 6.5 & 7.5 & 8.0 & 8.5 & 8.8 & 9.1 & 9.5 & 9.9 \\
\hline FANET & 3.5 & 3.8 & 4.1 & 4.8 & 5.0 & 5.2 & 5.8 & 7.1 & 8.5 & 9.0 \\
\hline
\end{tabular}

Table 5.6 Hello interval vs Average outage time (s) 


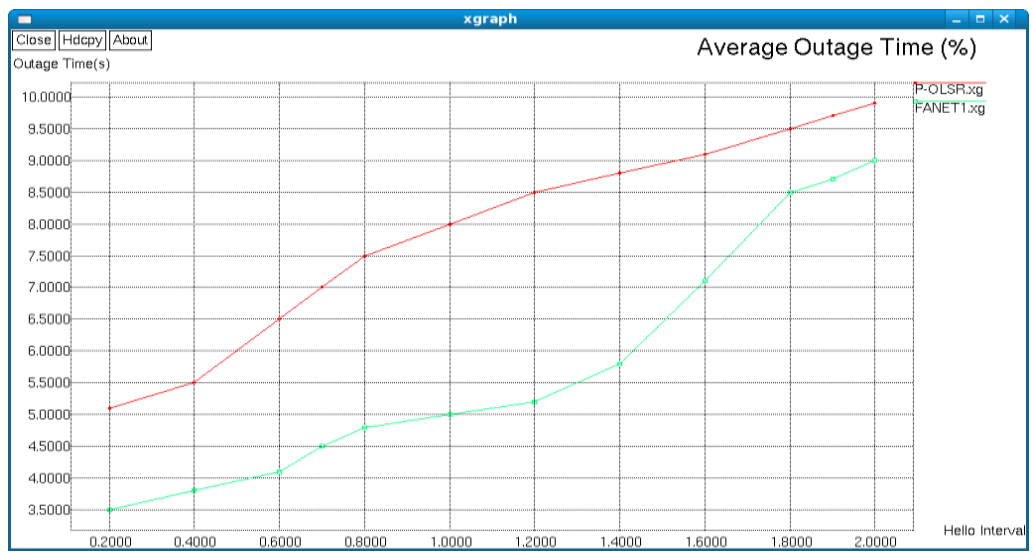

Figure 5.6 Hello interval vs Average outage time (s)

Fig. 5.6 show the output of connection between nodes vs outage time based on hello interval time. The FANET give fast make connection between nodes compare with Predictive OLSR.

\section{CONCLUSION}

The algorithm of FANET improves the packet delivery and reduce delay time between source to destination for UAVs network. In this approach, a node maintains the topology information involving its one-hop neighbors. The goal of this work is to optimize the network performance in a de-centralized manner, assuming that the relevant parameters such as the network topology and traffic demands are known at a central entity. Due to the high-mobility of the nodes, these networks are very dynamic and the existing routing protocols partly fail to provide a reliable communication. The FANET use connectivity of the speed and traffic aware routing algorithm to route the packet source to destination.

\section{REFERENCES}

[1] C. Barrado et al., "Wildfire monitoring using a mixed air-ground mobile network," IEEE Pervasive Comput., vol. 9, no. 4, pp. 24-32, Oct. 2010.

[2] Z. Sun et al., "BorderSense: Border patrol through advanced wireless sensor networks" Ad Hoc Netw., vol. 9, no. 3, pp. 468-477, May 2011. [Online]. Available: http://dx.doi.org/10.1016/j.adhoc.2010.09.008

[3] I. Rubin and R. Zhang, "Placement of UAVs as communication relays aiding mobile ad hoc wireless networks," in Proc. IEEE MILCOM, Oct. 2007, pp. 1-7.

[4] E. P. de Freitas et al., "UAV relay network to support WSN connectivity," in Proc. IEEE ICUMT, 2010, pp. 309-314. [Online]. Available: http://dx. doi.org/10.1109/ICUMT.2010.5676621

[5] F. Jiang and A. Swindlehurst, "Dynamic UAV relay positioning for the ground-to-air uplink," in Proc. IEEE GC Wkshps, Dec. 2010, pp. 17661770 .

[6] I. Bekmezci, O. K. Sahingoz, and S. Temel, "Flying ad-hoc networks (FANETs): A survey" Ad Hoc Networks, vol. 11, no. 3, pp. 1254-1270, May 2013. [Online]. Available: http://dx.doi.org/10.1016/j.adhoc.2012. 12.004

[7] O. Sahingoz, "Mobile networking with UAVs: Opportunities and challenges," in Proc. ICUAS, May 2013, pp. 933-941.

[8] K. Zhang, W. Zhang, and J.-Z. Zeng, "Preliminary study of routing and date integrity in mobile Ad Hoc UAV network," in Proc. ICACIA, Dec. 2008, pp. 347-350.

[9] J. Chroboczek, "The Babel routing protocol," Internet Eng. Task Force (IETF), Fremont, CA, USA, RFC 6126, Apr. 2011. [Online]. Available: http://www.rfc-editor.org/rfc/rfc6126.txt

[10] T. Clausen and P. Jacquet, "Optimized link state routing protocol (OLSR)," Internet Eng. Task Force (IETF), Fremont, CA, USA, RFC 3626, Oct. 2003. [Online]. Available: http://www.rfc-editor.org/rfc/rfc3626.txt

[11] E. W. Frew and T. X. Brown, "Networking issues for small unmanned aircraft systems," J. Intell. Robot. Syst., vol. 54, no. 1-3, pp. 21-37, Mar. 2009. [Online]. Available: http://dx.doi.org/10.1007/s10846-008-9253-2

[12] Y. Guo, X. Li, H. Yousefi'zadeh, and H. Jafarkhani, "UAV-aided cross-layer routing for MANETs," in Proc. IEEE WCNC, Apr. 2012, pp. $2928-2933$

[13] A. Alshbatat and L. Dong, "Cross layer design for mobile Ad-Hoc unmanned aerial vehicle communication networks," in Proc. ICNSC, Apr. 2010, pp. 331-336.

[14] M. Benzaid, P. Minet, and K. Al Agha, "Integrating fast mobility in the OLSR routing protocol," in Proc. 4th Int. Workshop Mobile Wireless Commun. Netw., 2002, pp. 217-221

[15] S. Rosati, K. Kru zelecki, L. Traynard, and B. Rimoldi, "Speed-aware routing for UAV Ad-Hoc networks," in Proc. 4th Int. IEEEWorkshop Wireless Netw. \& Control Unmanned Auton. Veh. Archit., Protocols Appl., 2013, pp. 1788-1792. 